\title{
A Broadband Metamaterial Absorber
}

\author{
Nikunj Goyal, Garima Tiwari
}

\begin{abstract}
This paper gives structure and standard execution of a broadband metamaterial protect at microwave frequencies. The unit cell of proposed structure combines of set-rectangular structure patches of copper put diagonally, at the most noteworthy purpose of the FR4 dielectric substrate and a steady ground plane of copper. The proposed shape demonstrates the broadband response of a maintenance exchange speed of two.7 $\mathrm{GHz}$ with more than 90 five\% absorptivity degree starting from 6.nine $\mathrm{GHz}$ to nine.6 GHz.It shows wideband ingestion upto forty five dimensions scene edges underneath underhanded recurrence for each TE and TM polarizations.
\end{abstract}

Index Terms:Metamaterial, Absorption.

\section{INTRODUCTION}

Metamaterial is phony substances that suggests odd electromagnetic wave (EM) characteristics that cannot be discovered in nature like poor refraction file, shielding conduct, radio wires, superlens, shields [1], and so forth at unique electromagnetic repeat. 'idealmetamaterial protect' had been proposed in 2008 with the aid of manner of Landy [1] seeing that than metamaterial shields have drawn noteworthy interests amongst various authorities. Because of its extremely-skinny thickness, lighter weight and prolonged sufficiency, these metamaterial shields are starting at now superseding the ordinary protections, which have the burdens of being massive and fragile.

Metamaterial defend is an incidental form and comprise a unit mobile.In standard, a metamaterial defend carries regular 3 layers of the MDM (metallic-dielectric - steel systems) kind of direction of movement, that is in discontinuous case of unit mobile has been comprehensively utilized in various plans. The ones structures can manage their remarkable electromagnetic parameters to such a degree, that the facts impedance of the structure finally ends up being immovably planned with the free area impedance. In the meantime, in case the lossy dielectric substrate ingests the occasion wave definitely, with the aid of then this outcomes in about brotherly love digestion. Up till this factor, numerous protect structures were arranged indicating one-of-a-kind homes, as an instance, single-band [1], multiband [5], statistics transmission up to date [4], broadband [2], polarization brutal [3] and significant-region ingestion [3] for numerous capability packages. In a part of the ones programs (like stealth improvement, anechoic chamber), broadband protections are significantly endorsed, even as in numerous packages, for instance, radar go-component decline, electromagnetic impedance/electromagnetic likeness affirmation and radio repeat recognizing verification, multi-band shields are frequently cherished.

Revised Manuscript Received on April 12, 2019

NikunjGoyal,ME scholar Dept of Electronics \& Communication, Jabalpur Engineering College, Jabalpur, Madhya Pradesh,India

GarimaTiwari,Assistant Professor, Dept of Electronics \& Communication, Jabalpur Engineering College, Jabalpur, Madhya Pradesh,India

\section{DESIGN AND REENACTMENT OF THE PROPOSED SHAPE:}

The top factor of view on the proposed form is showed up in determine 1.It well-knownshows a novel unit cellular which consists in rare manner involve metal patches set corner to nook backwards making rehash to each different.The patches appear to be set-sqaure circumstance of copper having conductivity of five.Eight x107 S/m with zero. $035 \mathrm{~mm}$ thickness. The bottom of the form is definitely of copper with thickness $0.035 \mathrm{~mm}$ and segregated from top layer with a dielectric substrate of FR-4 $(\varepsilon r=4.4$ and $\tan \delta=$ 0.02) of thickness $2 \mathrm{~mm}$. All of the additives of the unit mobile are improved as $a=10 \mathrm{~mm}, b=6 \mathrm{~mm}, c=8.6 \mathrm{~mm}$, $\mathrm{d}=2.3 \mathrm{~mm}, \mathrm{f}=2 \mathrm{~mm}, \mathrm{~g}=1.6 \mathrm{~mm}, \mathrm{w}=1 \mathrm{~mm}$.

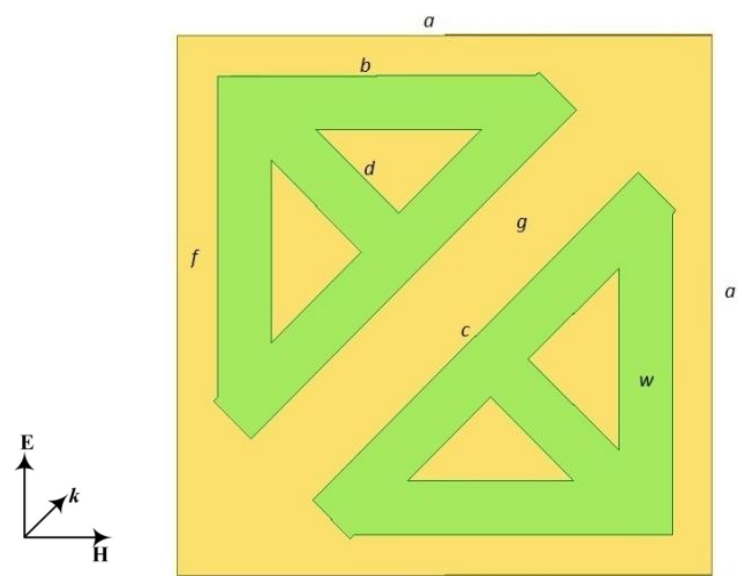

Figure.1 Unit cell of the proposed structure.

The absorbtion is excitedly related to the reflected photo and transmission and repeat of scene electromagnetic wave. a super metamaterial shield may be performed through constraining the reflected picture and transmission waves to get most noteworthy absorbtion. The transmission of 0 can be executed through using steel floor plane at the most insignificant of the structure, that is ordinarily thicker than the skin power of metal used on the assignment repeat. hence, when the EM waves are transmitted into the metamaterial protect, they will disappear because of the dielectric and metallic hardships.

The absorbtion can be resolved through Eqn (1),wherein $\omega$ is repeat of operation, $A(\omega)$ is the absorption, $R(\omega)$ is the reflected picture and $T(\omega)$ is the transmission and $S 11(\omega)$ and $S 21(\omega)$ are the relating S parameters. right legitimately here $\mathrm{Z} 0$ is free zone impedance and $\mathrm{Z}(\omega)$ is unit cell impedance.

$$
A(\omega)=1-R(\omega)-T(\omega)
$$




\section{A Broadband Metamaterial Absorber}

$$
A(\omega)=1-\left|S_{11}(\omega)\right|^{2}-\left|S_{21}(\omega)\right|^{2}
$$

The reflection of zero can be achieved by matching the impedance of the absorber with the impedance of free space through adjusting the geometric parameters of the structure to make the relative permeability $\mu_{r}$ and the relative permittivity $\mathcal{E}_{r}$ of the same value,

$$
\begin{gathered}
R(\omega)=\frac{Z(\omega)-Z_{0}}{Z(\omega)+Z_{0}} \\
Z_{0}=\sqrt{\mu_{0} / \varepsilon_{0}}=377 \Omega \\
Z(\omega)=\sqrt{\frac{\mu_{0} \mu_{r}(\omega)}{\varepsilon_{0} \varepsilon_{r}(\omega)}}
\end{gathered}
$$

The proposed structure is simulated in HFSS using periodic boundary conditions (master slave and floquet port) and the result of absorbtion is shown in Figure2.The proposed structure exhibits a broad bandwidth of $2.7 \mathrm{GHz}$ ranging from 6.9 to $9.6 \mathrm{GHz}$ with absorbtion of $95 \%$.

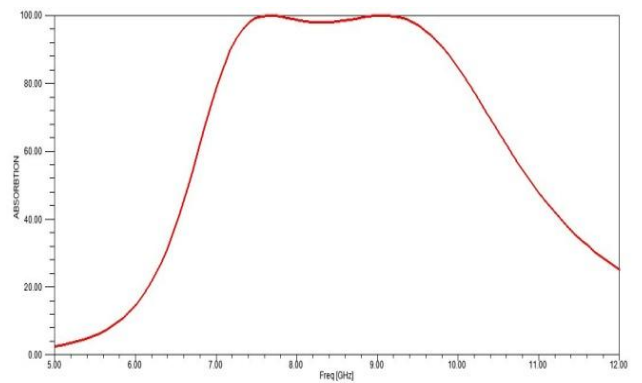

Figure 2.Simulated absorption performance of the proposed structure for the normal incidence EM wave.

\section{DISCUSSIONS AND RESULTS:}

The surface currents of proposed structure at the peak frequencies on the top and bottom surfaces are shown in figure 3 .
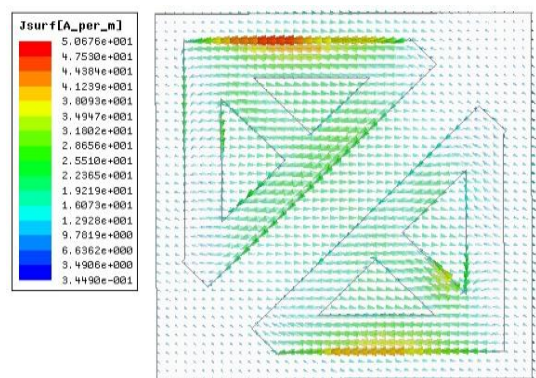

(a)

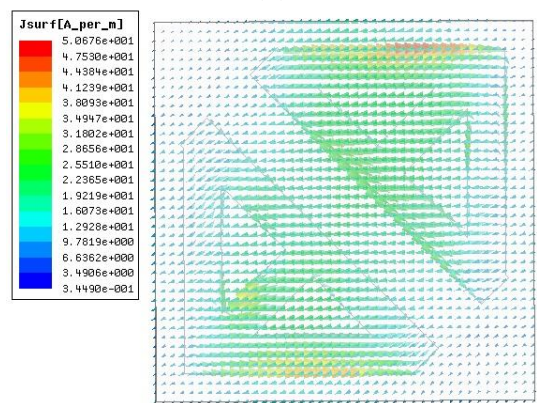

(b)

Figure 3. Surface current density distributions at the (a) top and (b) bottom surface of the proposed structure.
The counter parallel ground streams at the 2 steel fixes as obtrusive from figure 3 shape a round contemporary-day hover inside the substrate, that is obliged by means of using the event appealing subject, thusly making fascinating excitation.

The provoked electric fields inside the structure are in addition confirmed up in decide. Four at the frequencies of satisfaction, making electric excitation. The masking of these excitations results in strong electromagnetic protection.
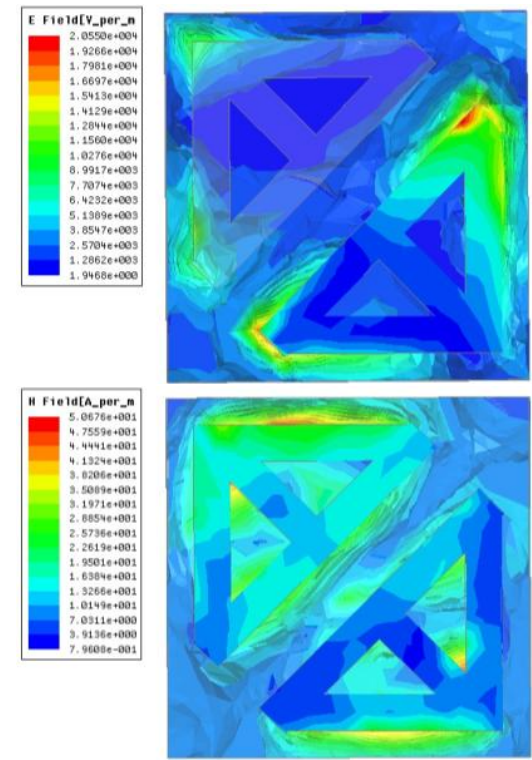

Figure. 4. Electric Field and magnetic Field distributions within the proposed structure

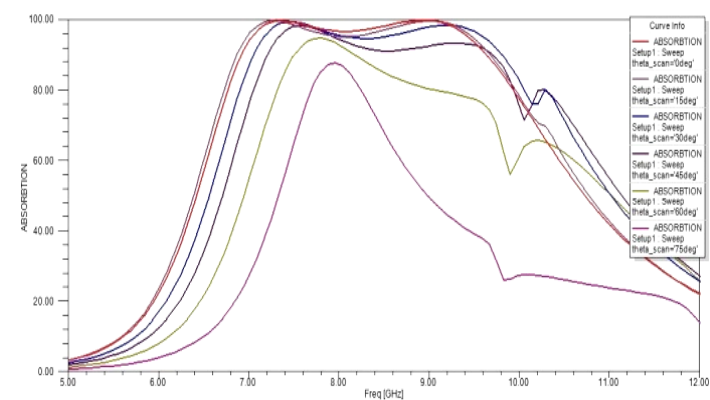

(a)

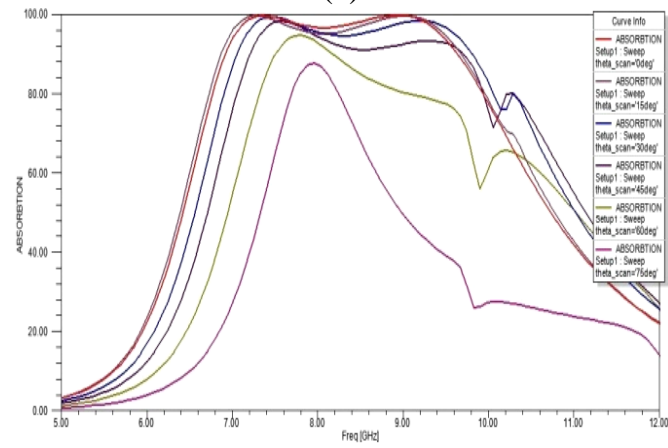

(b)

Figure 5. Simulated absorbtion response for the oblique incidence under (a) TE polarization and (b)TM polarization 
The shape is copied for one-of-a-type purposes of rate $(\theta)$ as exhibited in determine. five(a), underneath TE polarization. In this situation, the method for electrical locale is along X-rotate and the alluring spot and event wave vector headings are changed through method for a perspective $\theta$. The transmission limit of good estimated osmosis is kept up upto $45^{\circ}$ event perspective. The shape is in like manner considered for novel scene edges underneath TM polarization as plot in decide.five(b). authentic here, the course of alluring region is near to y-axis,and the electrical district and wave vector pieces of information are distinctive by methods for technique for a point of view $\theta$. Upto $45^{\circ}$ scene attitude, the basic ingestion exchange speed is discovered The proposed shape has in addition been concentrated for remarkable edges of polarization $(\varphi)$ as attested in watch 6 , wherein the method for event EMwave remains parallel to $\mathrm{z}$-course at the vague time as each the electric and alluring area make a point $\varphi$ with $\mathrm{x}$-course and $\mathrm{y}$-bearing independently.

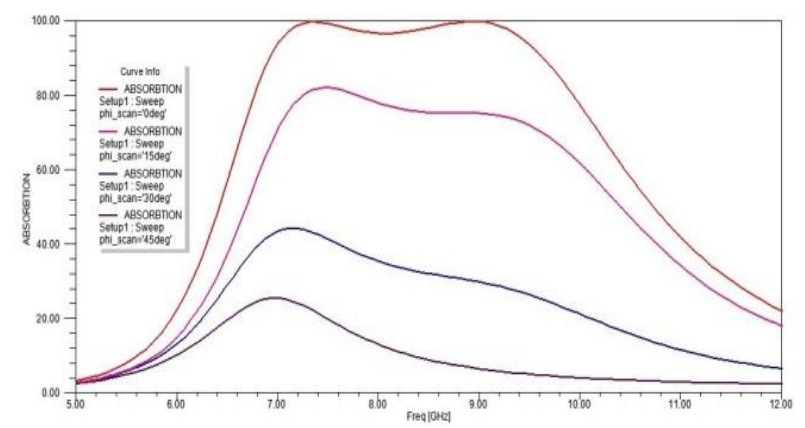

Figure.6. Simulated absorbtion response for the polarisation angle variation

The proposed shape have the 2 cowl symmetry along xy plane, the shape need to be regarded as simply upto $45^{\circ}$ edge of polarization. The reproduced ingestion response depicts in determine 6 , showing that the shape has broadband absorption upto forty five ${ }^{\circ}$ polarization part.

\section{CONCLUSION:}

A metamaterial defend has been analyzed with easy setsqaure framed patches made from copper placed corner to corner at the pinnacle surfaces. The proposed

Form indicates $2.7 \mathrm{GHz}$ ingestion facts transmission with over $905 \%$ absorptivity from 6 .Nine to nine.6 GHz. The shape is really $2.07 \mathrm{~mm}$ thick $(\sim \lambda / 15$ concerning inside frequency).The occupations of a couple of geometrical parameters of the form had been targeted to solve the broadband idea of the guard. The proposed shape shows substantial osmosis trade velocity upto forty five ${ }^{\circ}$ occasion plots for TE and TM polarizations as apparent from reproduced assessed responses.

\section{REFERENCES:}

1. Landy, N.I., Sajuyigbe, S., Mock, J.J., et al.: 'flawless metamaterial protect', Phys.Rev. Lett., 2008, a hundred, p. 207402.

2. Wang

Xin,ZhangBinzhen, WangWanjun, Wangjunlin,andDuanJunpin $\mathrm{g}$, et al. "shape and Characterization of a UltrabroadbandMetamaterial Microwave Absorber" IEEE Photonics mag, Vol. Nine, No. Three, June 2017.
3. S.Bhattacharya, S.Ghosh, D.Chaurasiya and V. Srivastava "A Broadband huge angle Metamaterial Absorber for safety applications" 2014 IEEE international Microwave and RF conference (IMaRC)

4. S.Bhattacharya, S.Ghosh, D.Chaurasiya and V. Srivastava " A extraordinarily-wideband ultra-moderate Metamaterial Absorber based totally on spherical split rings" DOI 10.1109/LAWP.2015.2396302, IEEE Antennas and wi-fi Propagation Letters.

5. Wang Xin, Zhang Binzhen, Wang Wanjun, Wang Junlin and DuanJunping "structure, introduction, depiction of a versatile twofold band metamaterial protect," IEEE Photonics mag, Vol. 9, No. 4, August 2017.

6. Bin Tang,YiqiZhu,XinZhou,LiHuang,Xiangzhong Lang "significant angle Polarization independent Broadband Absorber reliant on cocentric Multi split ring suggests" IEEE Photonics journal,Vol.9,No.6,December 2017.

7. SenfengLai, YanghuiWu,XiaoboZhu,WenhuaGu,Wen Wu "An Optically transparent Ultrabraodband Microwave Absorber" IEEE Photonics mag,Vol.Nine,No.6,December 2017. 